\title{
On the F-function of FEAL
}

\author{
Walter Fumy, Siemens AG \\ Systems Engineering Development, E STE 36 \\ D-8520 Erlangen, West Germany
}

\begin{abstract}
The cryptographic strength of a Feistel Cipher depends strongly on the properties of its $F$-function. Certain characteristics of the F-function of the Fast Data Encipherment Algorithm (FEAL) are investigated and compared to characteristics of the F-function of the Data Encryption Standard (DES). The effects of several straight-forward modifications of FEAL's F-function are discussed.
\end{abstract}

\section{Introduction}

A (cryptographic) function is called complete, if each of its output bits depends on every input bit [5]. A block cipher which is not complete, may be vulnerable to a known plaintext attack [1]. As the example of the Data Encryption Standard (DES, [7]) shows, the F-function of a Feistel Cipher needs not to be complete in order to ensure completeness of the block cipher itself. Despite the fact that each output bit of its F-function only depends on 6 input bits, the DES is complete after 5 rounds [6].

Due to the principle of a Feistel Cipher which operates on the two halves $L$ and $R$ of an input block, at least 3 rounds are necessary for its completeness. The internal states of a Feistel Cipher develop in the following way:

$\begin{array}{lll}\text { initial state: } & (\mathrm{L}, & \mathrm{R}) \\ \text { after round 1: } & (\mathrm{R}, & \left.\mathrm{L}+\mathrm{F}_{1}(\mathrm{R})\right) \\ \text { after round 2: } & \left(\mathrm{L}+\mathrm{F}_{1}(\mathrm{R}),\right. & \left.\mathrm{R}+\mathrm{F}_{2}\left(\mathrm{~L}+\mathrm{F}_{1}(\mathrm{R})\right)\right) \\ \text { after round 3: } & \left(\mathrm{R}+\mathrm{F}_{2}\left(\mathrm{~L}+\mathrm{F}_{1}(\mathrm{R})\right),\right. & \left.\mathrm{L}+\mathrm{F}_{1}(\mathrm{R})+\mathrm{F}_{3}\left(\mathrm{R}+\mathrm{F}_{2}\left(\mathrm{~L}+\mathrm{F}_{1}(\mathrm{R})\right)\right)\right)\end{array}$

A 3 round Feistel Cipher therefore is complete if $F_{2}$ is complete and if each of the output bits of $F_{3}$ depends on at least one of its input bits and each of the input bits of $F_{1}$ affects at least one of its output bits.

A function $f$ exhibits the avalanche effect, if an average of one half of its output bits change whenever a single input bit is complemented. Moreover, $f$ shows the strict avalanche criterion if each of its output bits changes with the probability of one half, when complementing one input bit [9]. The strict avalanche criterion includes the completeness of $f$. This property is considered essential for a "good" cryptographic transformation [9]. A random function will also exhibit the strict avalanche criterion.

The dependence matrix of a function $f: G F(2)^{n}-->G F(2)^{m}$ is a (nxm) matrix, whose entry $a_{i, j}$ gives the probability that the output bit $j$ of $f$ changes when its input bit $i$ is complemented. The function $f$ is complete if all elements in its dependence matrix have a nonzero value; it exhibits the strict avalanche criterion, if the value of every element is close to 0.5 . 


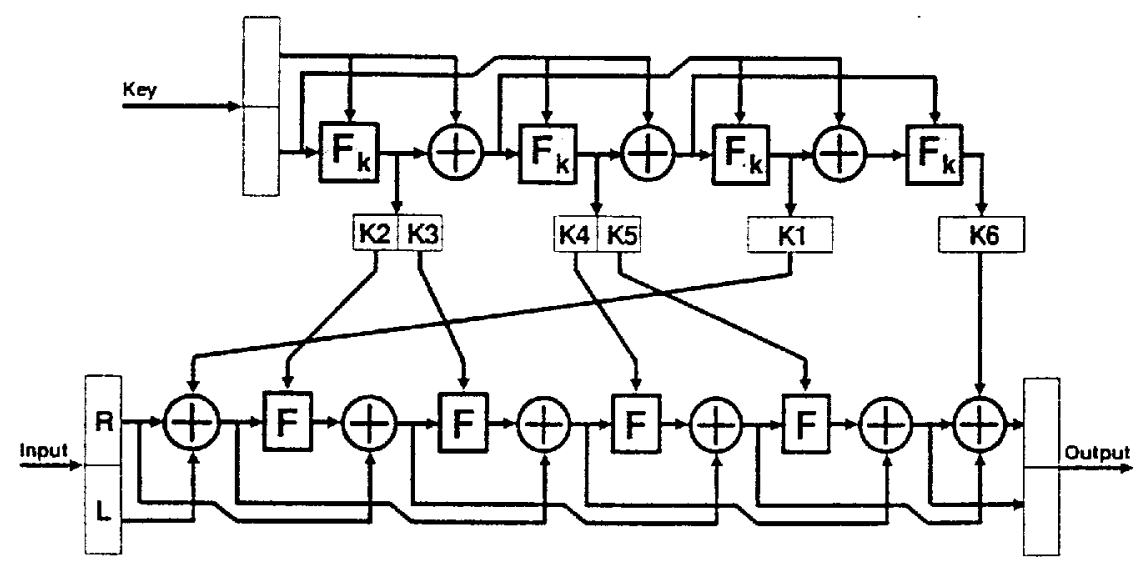

figure 1: Fast Data Encryption Algorithm (FEAL1)

\section{The Fast Data Encipherment Algorithm}

The Fast Data Encipherment Algorithm (FEAL) is a 6 round Feistel Cipher which operates on 64-bit blocks. Only 4 of its rounds make use of a non-trivial F-function (figure 1 shows FEAL1, [4], which slightly differs from the version given in [8]). The F-function of FEAL is shown in figure 2 . The function $F_{K}$ used for key expansion is similar to $F$. The structure of $F$ is byte-oriented. Its cryptographic strength depends on a non-linear S-function defined by
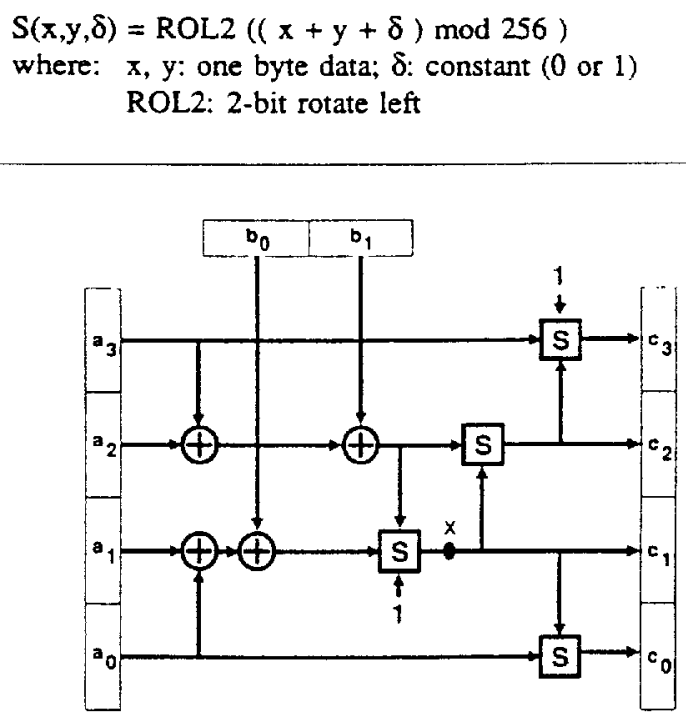

figure 2: F-function of FEAL

Analysis of the S-function reveals its incompleteness. Only one of its 8 output bits depends on every input bit. Moreover, its dependence matrix exhibits a very regular structure. Its upper half (which is identical to the lower half) is given below. 


$\begin{array}{cccccccc}1 / 64 & 1 / 128 & 1 & 1 / 2 & 1 / 4 & 1 / 8 & 1 / 16 & 1 / 32 \\ 1 / 32 & 1 / 64 & 0 & 1 & 1 / 2 & 1 / 4 & 1 / 8 & 1 / 16 \\ 1 / 16 & 1 / 32 & 0 & 0 & 1 & 1 / 2 & 1 / 4 & 1 / 8 \\ 1 / 8 & 1 / 16 & 0 & 0 & 0 & 1 & 1 / 2 & 1 / 4 \\ 1 / 4 & 1 / 8 & 0 & 0 & 0 & 0 & 1 & 1 / 2 \\ 1 / 2 & 1 / 4 & 0 & 0 & 0 & 0 & 0 & 1 \\ 1 & 1 / 2 & 0 & 0 & 0 & 0 & 0 & 0 \\ 0 & 1 & 0 & 0 & 0 & 0 & 0 & 0\end{array}$

Since the S-function is not complete, the F-function of FEAL can not be complete. An analysis of the powers of $F$ results in $F^{i}$ being complete for $i>1$ and exhibiting the strict avalanche criterion for $i>2$ (see figure 4). In this respect the F-function of FEAL does better than the F-function of DES where $\mathrm{F}^{j}$ is complete for $j>2$ and shows the strict avalanche criterion for $j>3$ (see also figure 4). For this reason FEAL is complete after 4 rounds whereas DES needs 5 rounds in order to become a complete block cipher (see figure 3 ).

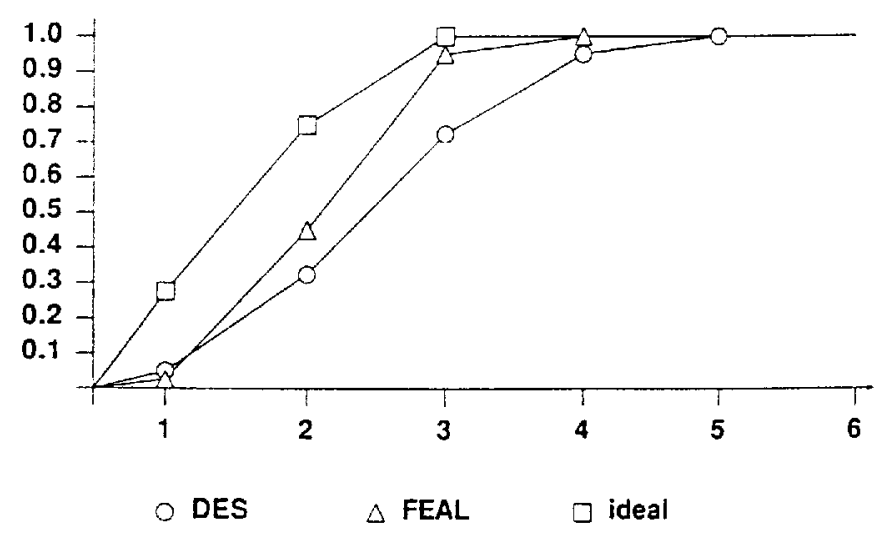

figure 3: Increasing completeness of DES and FEAL

\section{Modifications of FEAL's F-function}

There are straight-forward modifications of FEAL's F-function that will further improve the properties discussed above. Moreover, these modifications allow the introduction of a non-linear function that can be chosen at random and will therefore overcome any distrust of the S-functions.

D.Davies has suggested to modify FEAL's F-function by replacing the operation ROL2 by an 8-bit Sbox [2]. By this modification 4 (possibly different) S-boxes are added to the F-function. Although a randomly chosen S-Box will show the strict avalanche criterion, the modified F-function will not exhibit the same property. It is complete, however, and $\mathrm{F}^{\mathrm{i}}$ will show the strict avalanche criterion for $\mathrm{i}>1$ (see figure 4).

We suggest a modification that uses only one additional 8-bit S-Box in the position marked by $X$ in figure 2. The input of this S-Box depends on every byte of the input of F. Its output affects every byte of the output of $F$. The effect of this modification is similar to the effect of the modification discussed above. The modified $F$-function is complete and $F^{i}$ will exhibit the strict avalanche criterion for $\mathrm{i}>1$ (see figure 4).

Both modifications of FEAL's F-function will counteract den Boer's cryptanalysis of this cipher [3]. 


\begin{tabular}{|l|l|l|l|l|l|}
\cline { 3 - 6 } \multicolumn{2}{l|}{} & $\mathbf{F}$ & $\mathbf{F}^{2}$ & $\mathbf{F}^{3}$ & $\mathbf{F}^{4}$ \\
\hline DES & dependence & 0.188 & 0.875 & 1.000 & 1.000 \\
& mean & 0.119 & 0.375 & 0.493 & 0.500 \\
& variance & 0.063 & 0.027 & 0.00053 & 0.00026 \\
\hline FEAL & dependence & 0.883 & 1.000 & 1.000 & 1.000 \\
& mean & 0.308 & 0.481 & 0.500 & 0.500 \\
& variance & 0.112 & 0.013 & $\mathbf{0 . 0 0 0 2 6}$ & $\mathbf{0 . 0 0 0 2 4}$ \\
\hline FEAL + 1 & dependence & 1.000 & 1.000 & 1.000 & 1.000 \\
S-Box & mean & 0.505 & $\mathbf{0 . 4 9 9}$ & 0.500 & 0.500 \\
& variance & 0.00096 & 0.00024 & 0.00026 & 0.00026 \\
\hline FEAL + 4 & dependence & 1.000 & 1.000 & 1.000 & 1.000 \\
S-Boxes & mean & 0.503 & $\mathbf{0 . 5 0 1}$ & $\mathbf{0 . 5 0 0}$ & $\mathbf{0 . 5 0 0}$ \\
& variance & 0.00054 & $\mathbf{0 . 0 0 0 2 5}$ & $\mathbf{0 . 0 0 0 2 6}$ & $\mathbf{0 . 0 0 0 2 4}$ \\
\hline
\end{tabular}

figure 4: Comparison of different F-functions

\section{References}

[1] Chaum, D.; Evertse, J.-H.: Crypranalysis of DES with a Reduced Number of Rounds, in: Advances in Cryptology - Crypto '85, H.C.Williams ed,

Lecture Notes in Computer Science, 218 (1986), 192-211

[2] Davies, D.W.:

private communication (1987)

[3] den Boer, B.: Cryptanalysis of FEAL, presented at Crypto 87

[4] ISO: Introduction to a New Encipherment Algorithm FEAL. ISO/TC97/SC20/WG1 N36 (1985)

[5] Kam, J.B.; Davida, G.1.: Structured Design of Substitution-Permusation Encryption Nerworks, IEEE Trans. Computers, 28 (1979), 747-753

[6] Meyer, C.H.; Matyas, S.M.: Cryptography: A New Dimension in Computer Data Security, (John Wiley \& Sons, New York, 1982)

[7] National Bureau of Standards: Data Encryption Standard, FIPS Publ. 46, Washington D.C., 1977

[8] Shimizu, A.; Miyaguchi. S.: Fast Data Encipherment Algorithm FEAL. presented at Eurocrypt 1987

[9] Webster, A.F.; Tavares, S.E.: On the Design of S-Boxes, in: Advances in Cryptology - Crypto 85, H.C.Williams ed, Lecture Notes in Computer Science, 218 (1986), 523-534 